\title{
Applying theories for using non-monetary incentives for citizens to participate in crowdsensing projects
}

\author{
Brenda Scholtz, Clara Mloza-Banda \\ Nelson Mandela University, Port Elizabeth, South Africa
}

\begin{abstract}
In recent years crowdsensing has become a hot topic amongst researchers. Crowdsensing can incentivise and empower citizens to use their mobile phones to collect and share sensed data from their surrounding environments. The purpose of this paper is to report on the application of the incentive theory and the Theory of Planned Behaviour (TPB) as a lens from which to investigate the non-monetary incentives and participation profiles (intentions and motivations) of citizens from around the world, who could participate in a crowdsensing project for water resource monitoring (WRM). The conceptual framework was used in a survey of citizens. The findings revealed that TPB can be successfully used for predicting behavioural intentions and classified several types of motivational factors for participation in crowdsensing projects for WRM. Guidelines for crowdsensing projects are provided that can improve the success rate of WRM projects.
\end{abstract}

Keywords: crowdsensing, water resource monitoring, theory of planned behaviour, data collection protocols

Categories: • Information systems $\sim$ Collaborative and social computing systems and tools • Human-centred computing $\sim$ Empirical studies in ubiquitous and mobile computing

Email:

Brenda Scholtz brenda.scholtz@mandela.ac.za (CORRESPONDING)

\section{Article history:}

Received: 21 Jun 2019

Accepted: 16 Oct 2019

Available online: 20 Dec 2019

\section{INTRODUCTION}

Goal 6 of the United Nations' Sustainable Development Goals (SDGs) strives to "ensure availability and sustainable management of water and sanitation for all" (Economic and Social Council, 2018). One target of this goal is to support and strengthen local communities to participate in water, sanitation and hygiene (WASH) project implementations $s$ in order to ensure their success and sustainability (Hall et al., 2016). In Sub-Saharan Africa, approximately 130 million citizens do not have access to safe water but have mobile network coverage (Nique \& Opala, 2014). Water resource monitoring (WRM) is described as the collection of data that reflects the existing status quo of water resources at certain points and time intervals, in order to manage the infrastructure of water resources and services. One important factor to provide sound water supply and accurate

Scholtz, B. and Mloza-Banda, C. (2019). Applying theories for using non-monetary incentives for citizens to participate in crowdsensing projects. South African Computer Journal 31(2), 99-116. https://doi.org/10.18489/sacj.v31i2.787 Copyright (C) the author(s); published under a Creative Commons NonCommercial 4.0 License (CC BY-NC 4.0).

$S A C J$ is a publication of the South African Institute of Computer Scientists and Information Technologists. ISSN 1015-7999 (print) ISSN 2313-7835 (online). 
sanitation services is the availability of accurate and consistent information (Rivett, Gool, Champanis \& Nyemba-Mudenda, 2014). Therefore the use of pervasive and ubiquitous technologies such as mobile devices for information dissemination has increased in popularity in the water sector (Nel, Booysen \& van der Merwe, 2014). The affixing of a sensory device to a mobile phone provides the opportunity to track dynamic information about humans, the environment and to understand their patterns. In crowdsensing, a mobile phone acts as a sensor, collecting, processing and distributing data (Yang, Xue, Fang \& Tang, 2015).

Crowdsensing can potentially provide practitioners, researchers and other stakeholders with the ability to solicit data collection and crowd wisdom over large geographical areas by the connection of a large number of people at once (Yadav et al., 2013). High-end mobile phones on the market today have general purpose sensors such as cameras and microphones as well as specialised sensors including GPS, ambient light sensors, digital compasses, proximity sensors and accelerometers. Due to the advent of mobile devices, citizens can act as sensors and actively participate in collecting data used to improve the actions of the organisation responsible for water sanitation (Gao, Li, Zhao, Fan \& Han, 2015).

An analysis of the literature revealed that many relevant studies on crowdsourcing have been conducted, for example Guo, Yu, Zhou and Zhang (2014), Hung, Tam, Tran and Aberer (2013), Lowry and Fienen (2013), Massung, Coyle, Cater, Jay and Preist (2013). Some of these, such as (Massung et al., 2013) and (Hung et al., 2013), focused on crowdsourcing in the context of environmental improvement. Other studies Han, Graham, Vassallo and Estrin (2011), He, Chan and Guizani (2015), Lendák (2016), Liu, Shen and Zhang (2016), Pankratius, Lind, Coster, Erickson and Semeter (2014) focused on techniques for crowdsensing whilst Hutchings et al. (2012) investigated privacy issues of crowdsensing. A study of mobile phone applications for solving WASH issues was conducted by Minkman (2015). Studies such as Ogie (2016) and Wilson (1997) investigated motivational factors for participation in citizen science but only a few studies Jaimes, Vergara-Laurens and Raij (2015), Kanhere (2011), Rotman, Hammock, Preece, Hansen and Boston (2014) were found on incentives and participation profiles for crowdsensing. Rotman et al. (2014) reviewed a variety of incentives that motivate people to contribute to a crowdsensing project. They proposed a taxonomy of crowdsensing incentive mechanisms and highlighted a need for further investigation of other types of incentives and studies on citizen profiling. In crowdsensing initiatives citizens are the primary actors; therefore the usefulness and performance of the crowdsensing system depends on the citizens' intentions or willingness to participate in the collection of data. There is little understanding of what incentivises or prohibits citizens from participating in crowdsensing.

The purpose of this paper is

1. to investigate the profile of participants regarding their intention to participate in a crowdsensing for WRM and

2. to identify and classify the key motivational factors for participation in crowdsensing in WRM.

The research reported on forms part of a larger research study that aims to design a comprehensive method for crowdsensing projects. The study conducted a critical review of related literature and 
used the Theory of Planned Behaviour (TPB), incentive theory and a motivational framework for crowdsensing as theories to undergird the research. A survey of citizens was then conducted using the theoretical framework to guide the questionnaire design. The findings provide a valuable theoretical and practical contribution, since they can lead to a deeper understanding of the participant profile for WRM by researchers. Practitioners can use this knowledge to guide project coordination and for data collection protocols that can improve the success of crowdsensing for WRM.

The rest of this paper is organised as follows: The next section presents an overview of crowdsensing, the motivational factors and incentives for crowdsensing and the TPB theory. Section 3 explains the research design process followed in the analysis of citizens' intentions and motivations. In Section 4, the survey results are analysed and presented and several success factors for successful crowdsensing are made. The final section draws several conclusions and outlines future research directions.

\section{LITERATURE REVIEW}

\subsection{Incentive theory and crowdsensing}

A smart community has been described as a geographical area ranging in size from a neighbourhood to a country whose residents, organisations and governing institutions are using IT to transform their region in significant ways (Liu et al., 2016; Traverso, 2015). The success of a smart community is reliant on the proactive participation of the citizens living in the area to improve their quality of life (Cilliers, Flowerday \& McLean, 2016). ICT infrastructure and applications are important but without the citizens' engagement and willingness to collaborate and cooperate with external stakeholders, there is no smart community (Traverso, 2015). Crowdsensing is an approach to developing a smart community that has gained increasing popularity in recent years (Gong \& Shroff, 2017; Liu et al., 2016).

Crowdsensing is based on the crowdsourcing concept of obtaining the needed services or content by soliciting contributions from people (Han et al., 2011). With crowdsensing, citizens can act as sensors, to sense and interpret their surroundings and provide information about this interpretation through text, voice, video, location and other means of communication (Gao et al., 2015). Citizens can use their mobile device's camera as a video and/or image sensor, the microphone as an acoustic sensor and GPS receivers to provide location information (Jaimes et al., 2015; Nel et al., 2014). The collected data, stored in a central database server, can be directly accessed or further processed to create useful and relevant information for stakeholders (Hutchings et al., 2012). Crowdsensing provides researchers and organisations the opportunity to solicit the "wisdom of the crowd" from users of mobile devices and for collection and analysis of crowd-powered data over wide geographical areas by connecting to a vast number of people at once (Bozzon, Houtkamp, Kresin, de Sena \& de Weerdt, 2015; Han et al., 2011).

The recruitment, motivation and retention of participants is always a significant challenge for crowdsensing (Bosha, Cilliers \& Flowerday, 2017). An incentive theory for motivating participation in a crowdsourcing project was proposed by Killeen (1982). Incentives can be described as a type of 
motivation that encourages people to do their best at a particular task (Gassenheimer, Siguaw \& Hunter, 2013). The incentives for participating in crowdsensing may differ depending on the task to be performed and can be classified according to three types: intrinsic, extrinsic or internalisedextrinsic.

Intrinsic incentives provide the internal feelings or emotion that results in satisfactorily completing a task (Hung et al., 2013). Examples of intrinsic incentives are satisfaction, interest and enjoyment. Extrinsic incentives include both monetary and tangible non-monetary incentives that motivate citizens to contribute data (Gassenheimer et al., 2013; Killeen, 1982). The incentive theory was extended by adding internalised-extrinsic incentives to motivate citizens to provide data contributions (Killeen, 1982) to improve their standing in their community, and to teach and influence other members of the community (Wiggins, 2011). The three categories of incentives can be further classified into five sub-categories of motivation (see Figure 1) that encourage or motivate users to participate in crowdsensing (Rotman et al., 2014; Wilson, 1997), namely: collective; intrinsic motivation; social interaction; self-benefit; and self-efficacy.

Collective, intrinsic motivation, social interaction and self-benefit incentives were identified by Rotman et al. (2014) as the key factors affecting the level of participation in crowdsensing projects. Collective incentives encourage citizens to work together to improve their communities and society for the greater good, social responsibility and/or conservation (Rotman et al., 2014; Wilson, 1997). Citizens can also be involved in crowdsensing projects based on their own intrinsic motivation (Nevid, 2013). Intrinsic incentives allow participants to volunteer due to their inherent interest, enjoyment or leisure. Citizens may also be motivated to participate in crowdsensing for social interaction such as peer feedback, reinforcing others and to interact with other citizens using technologies such as social networks, blogs and SMS (Lendák, 2016; Rotman et al., 2014). Furthermore, self-benefit incentives allow participants of crowdsensing projects to receive gratification for their data contributions, in the form of system feedback, self-promotion or furthering the participants' opportunities (Rotman et al., 2014). On the other hand, self-efficacy incentives motivate citizens to participate in crowdsensing to generate scientific knowledge or to be part of scientific work (Wilson, 1997).

While an incentive can be described as a reward, reinforcement or external stimulus that motivates an individual to perform a behavior (Gassenheimer et al., 2013), motivational factors are the wants and needs that drive a certain behaviour and can activate, direct, and sustain a behaviour (Geoghegan, Dyke, Pateman, West \& Everett, 2016). In this regard, crowdsensing project coordinators need to understand what factors can motivate citizens to participate in crowdsensing.

\subsection{A framework of motivational factors for crowdsensing}

As a foundation, this study used Minkman's framework (Ogie, 2016) of more than 40 citizen motivational factors that can motivate citizens to make the first step toward participation in crowdsensing. However, motivational factors related to financial compensation were were omitted from the adopted framework as they are not relevant to the purposes of this study, which only explores non-monetary incentives. The categories illustrated in Figure 1 were used to classify the remaining motivational factors of crowdsensing provided in (Ogie, 2016). Three additional factors were added to the 


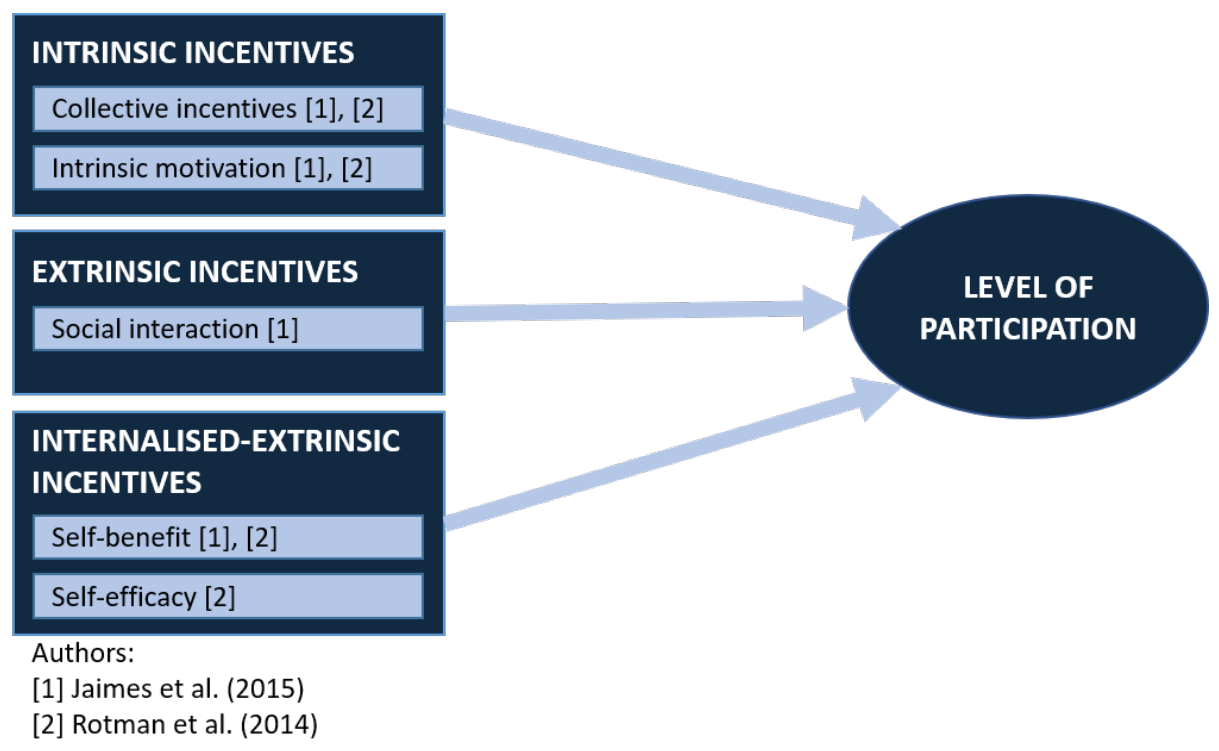

Figure 1: Incentives for crowdsensing

framework as suggested by Ajzen (1991). These factors are: help a specific site (intrinsic); get some exercise (self-benefit); and influenced by someone (self-benefit).

The resulting framework includes 25 motivational factors that relate to the initial engagement in a crowdsensing project. These factors do not consider the long-term participation of volunteers. For this reason the framework incorporates the five motivational factors recommended by Wilson (1997) that encourage long-term participation in crowdsensing projects, namely:

- trust: A volunteer may maintain trust in the process, the usage of their data, value and leadership roles.

- acknowledgement and attribution: Volunteers would like to be recognised and attributed for the work they perform and see that the work is bringing value to the area of concern.

- policy and activism: Volunteers would want to see the impact of their work on the government, institutions or community depending on the project objectives.

- mentorship: Other volunteers may seek deeper involvement in the project.

- common goals: A volunteer may remain in a project long-term because it aligns with their common goals.

In addition to the 30 motivational factors identified in the framework, designers of crowdsensing systems also need to consider demotivational factors. Yadav et al. (2013) state that a user would not be interested in participating in crowdsensing unless it receives a satisfying reward to compensate its 
resource consumption and potential privacy breach. Citizens participating in crowdsensing consume their own resources such as computing and battery power (Yadav et al., 2013). In addition, they expose themselves to potential privacy threats by sharing their sensed data with location tags. Other demotivational factors are, for example, that citizens believe the data won't be used or they lack confidence in their abilities to collect the required data (Rutten, Minkman \& van der Sanden, 2017). The framework of motivational factors (initial and long-term) illustrated in Figure 2 incorporates the seven demotivational factors (or barriers) proposed by Yadav et al. (2013).

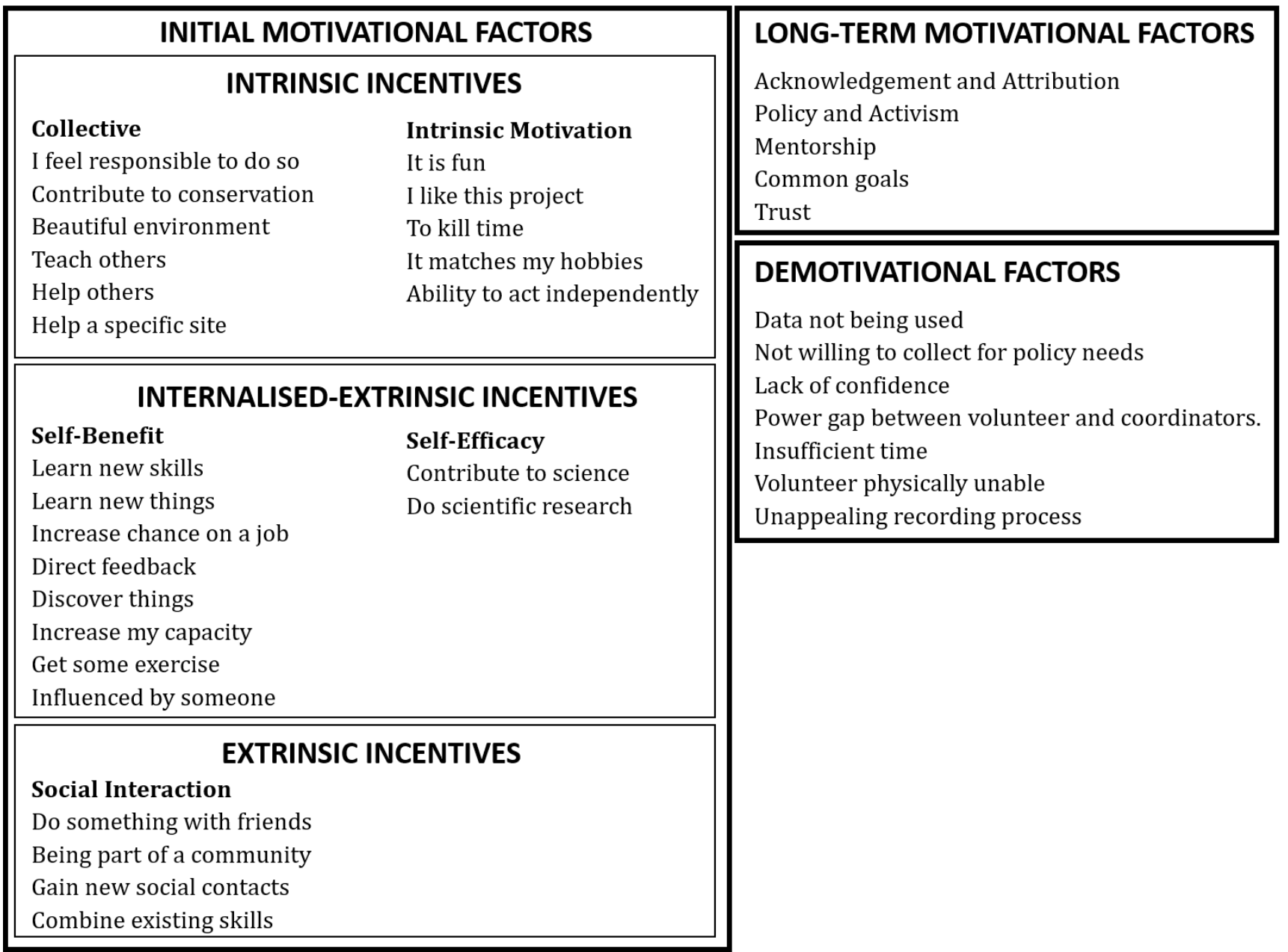

Figure 2: Framework of motivational factors for crowdsensing

\subsection{Theory of Planned Behaviour}

Crowdsensing requires continuous data collection to be successful (Rotman et al., 2014). The continuous submission of data by participants can be defined as a behaviour. TPB is a commonly- 
used theory first developed by Fishbein and Ajzen (2011) that allows investigation into specific behaviours. The TPB states that behavioural intention (BI) "is the key predictor of behaviour, with behavioural attitudes, subjective norms and perceived behavioural control as the most important antecedents of a person's intention to perform the behaviour" (de Montalvo, 2003; Fishbein \& Ajzen, 2011). Behavioural attitude (BA) refers to a person's evaluation of the behaviour which includes beliefs about the consequences as well as their positive and negative judgements about performing the behaviour.

Typically, the more positive an individual's attitude is toward a certain behaviour, the higher the chance they will engage in the behaviour. Subjective norms (SN) refer to a person's estimation of the social pressure to perform the target behaviour or not. SNs are the beliefs about how other people expect the person to behave and how the person feels about their expectation. Perceived behavioural control (PBC) explores how much control a person has over their own behaviour and their confidence in being able to perform the behaviour. Great PBC and stronger positive social pressure are also seen to increase the chance of an individual performing the behaviour.

\section{RESEARCH DESIGN}

This research aims to address two research objectives:

RO1: Explore the relationship between behavioural attitude, subjective norms and perceived behavioural control towards taking part in WRM and how the constructs influence the intention to participate.

RO2: Investigate the key motivational factors for participation in crowdsensing in WRM to fuel the design of appropriate incentive mechanisms that allow continuous collection of data.

Following the work of previous researchers (Gharesifard \& Wehn, 2015; Martin, Weiler, Reis, Dimmock \& Scherrer, 2017) who applied TPB to study spatial data sharing behaviour, this study utilised TPB to undergird the research and to predict the intention or willingness of citizens to collect data about water resources (Figure 3). The appropriate application of TPB can predict the intention to perform a behaviour (Joachim, Kamarudin, Aliagha \& Ufere, 2015). As illustrated in Figure 3, the null hypothesis of our study to address RO1 is:

$H_{0}$ : Behavioural intentions (BI) to make water data submissions are not influenced by behavioural attitude (BA), subjective norms (SN) or perceived behavioural control (PBC).

However, having an intention does not confirm the engagement of a behaviour (Joachim et al., 2015). A researcher seeking behaviour change needs to discover what emotional, social or cognitive forces activate the behaviour (Harpe, 2015). Those forces, whether internal or external, must be able to induce an individual's motivation and stimulate their desire and energy to be continually interested and committed to performing a given behaviour. Thus, the second objective of the study was to investigate the motivational factors for participation in WRM as well as demotivational factors that would make it difficult to participate. 


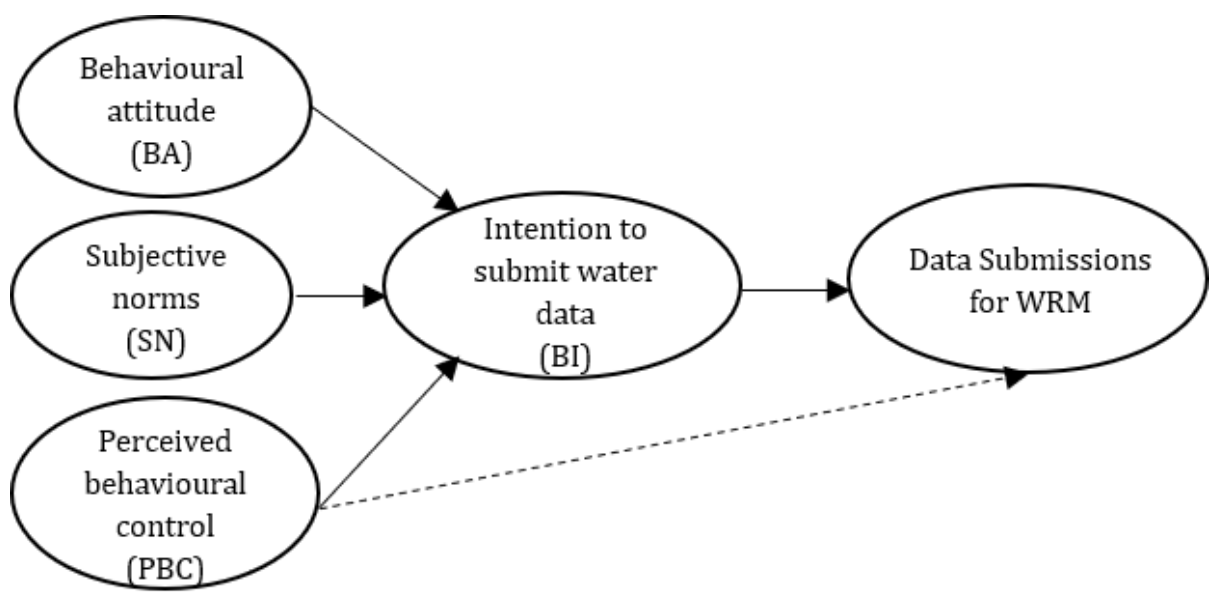

Figure 3: Conceptual model for submissions of water data using TPB

A quantitative empirical research approach was used to investigate citizens' intentions and motivations for participating in WRM, specifically for contributing data on water pollution and water wastage (e.g. littering, dirty water, water leaks, burst pipes and tanks) within the general public. A survey was conducted using an online questionnaire. The first part of the questionnaire, aimed at addressing RO1, was guided by TPB (Fishbein \& Ajzen, 2011) to understand citizens' intentions to participate in WRM. The questions in the first section of the questionnaire were designed on the conceptual model of TPB for water data submissions as illustrated in Figure 3.

A 5-point Likert scale was used in this section for the closed-ended questions. The scale ranged from 1 to 5, where 1 represents a negative response and 5 represents a positive response. Based on recommendations by Jiménez and Pérez-Foguet (2010), statistical ranges were applied to the data from the Likert scale question responses to categorise the responses as negative [ 1 to 2.6), neutral [2.6 to 3.4] and positive (3.4 to 5]. A pilot study was carried out before deploying the questionnaire to identify issues with the instrument and find possible solutions. The pilot study involved five individuals and resulted in minor changes being made to the questionnaire. Data analysis was done in Microsoft Excel using the built-in Analysis Toolpak.

The second part of the questionnaire, aimed at addressing RO2, was guided by incentive theory and the theoretical framework of factors affecting citizens' motivations to participate in crowdsensing (Figure 3). An understanding of these two elements of a citizen's profile can assist with designing appropriate incentive mechanisms in crowdsensing systems. Snowball sampling (Baltar \& Brunet, 2012) was used and the survey link was sent to several researchers, social media influencers, government agents and citizens. These individuals were asked to distribute the link through their mailing lists and social networks (LinkedIn, Facebook and Twitter). A link to the survey was also posted on several sites including Poll-Pool.com, SurveySwap.io, App.SwapSurvey.com, SurveyCircle.com to access individuals in other parts of the world. Furthermore, the sample size was extended by asking each respondent if they knew anyone else (online or offline contacts) who could meet the sample 
criteria (anyone over the age of 18) and participate in the study. The survey was online for five weeks.

\section{FINDINGS AND RECOMMENDATIONS}

\subsection{Profile of respondents}

Of the 123 responses obtained from the survey, three were incomplete and had to be removed, leaving 120 valid responses. Nearly a quarter (22.5\%) of the respondents were from developed countries (USA, United Kingdom, Australia, Netherlands, Germany and Canada) while 77.5\% were from developing countries (South Africa, Malawi, Benin, Uganda, Kenya and Swaziland).

\subsection{Intent to submit data}

TPB measures the generalised intention to perform a behaviour, in this case the intention to submit water data (BI). TPB uses three variables to measure BI and these were converted to statements, where the respondents were asked to provide individual ratings of whether they (1) expect, (2) want and (3) intend to submit water data. A Pearson correlation test was performed to calculate the relationship between the three variables. The findings showed that the Pearson's correlation ranged from 0.744 to 0.778 , which Larose (2006) consider as strong positive relationships. For this reason, the overall mean of the three variables can be used to accurately represent their BI.

The mean BI across all respondents was 3.97 (std: 1.00), which is a positive rating. The frequency distribution across all respondents $(n=120)$ over five groupings is shown in Figure 4. A majority of the respondents $(n=84)$ showed a positive response to participating in WRM, seen in the range $(3.4$ to 5]. Only 11 respondents had a negative response, [ 1 to 2.6) and 25 respondents had a neutral response, [2.6 to 3.4$]$.

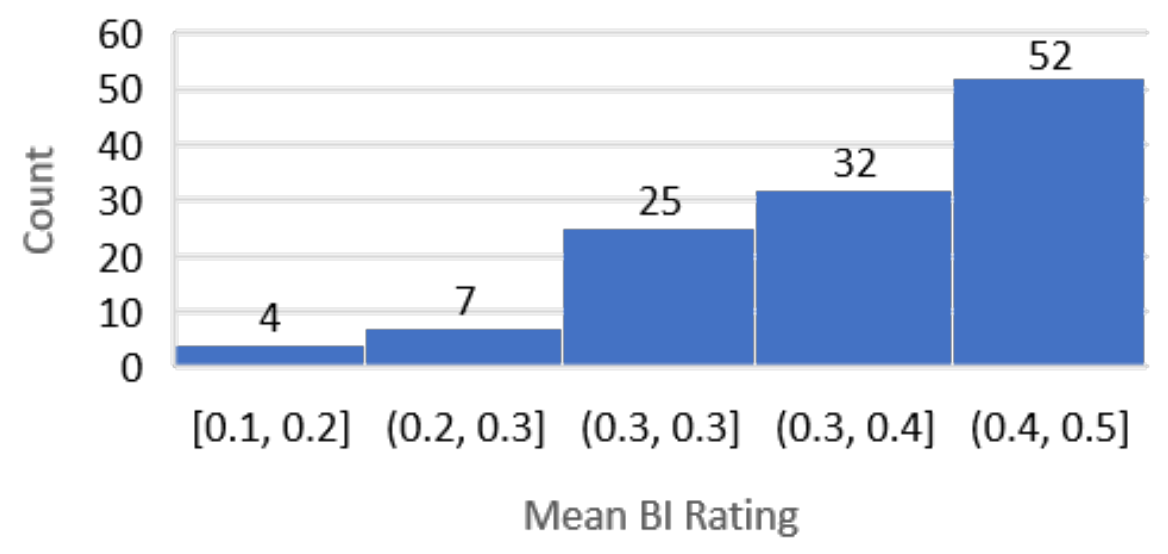

Figure 4: Distribution of mean BI ratings across all respondents $(n=120)$ 
Table 1: Pearson correlation matrix of all variables related to BI $(n=120)$

\begin{tabular}{rrrrr}
\hline & BI & BA & SN & PBC \\
\hline BI & 1 & & & \\
BA & $0.5648 *$ & 1 & & \\
SN & $0.5717 *$ & 0.3697 & 1 & \\
PBC & $0.4310 *$ & 0.3292 & 0.1449 & 1 \\
\hline
\end{tabular}

An analysis of mean ratings for BI for respondents from developing versus developing countries were also conducted. Respondents from developing countries were positive $(\mu=4.14$, std. $=0.91)$ towards the intention to participate in WRM, while respondents from developed countries had a neutral mean intention $(\mu=3.40$, std. $=1.08$ ). The positive rating towards water data sharing in developing countries could possibly be attributed to the lack of significant improvement in service delivery they experience as reported by Rivett et al. (2014). Developing countries face a lack of shared and relevant information between communities and service providers (Glotzbach, Barakzai, Adisu \& Tiwari, 2013; Sarstedt, Ringle, Smith, Reams \& Hair, 2014). A third of water sources in rural areas are non-functional and require servicing, thus there is an urgent need for improvement in the sharing of relevant information between communities and service providers in developing countries.

\subsection{Prediction of intention using TPB variables}

The Pearson's correlation between the four variables (BI, BA, SN and PBC) was calculated to express the degree of correlation between the variables. The results (Table 1) show that as BA, SN and $\mathrm{PBC}$ increase, the individual's intention to participate (BI) also increases. The Pearson correlation between BI and each of the other variables (0.43 to 0.56) show strong relationships (Larose, 2006).

Regression analysis was used to measure the extent to which BI is significantly associated with the three explanatory variables: BA, SN and PBC (Table 2). Only 53\% of the variation in intentions $(r 2)$ is explained by the independent variables BA, SN and PBC. However, the interpretation of $r 2$ is subjective depending on the field, and low $r 2$ values (such as 0.25 ) are not unusual in social sciences due to person-to-person variability (Braun \& Clarke, 2006). Thus, due to the r2 value of 0.53 , it can be concluded that each of the three variables (BA, SN and PBC) influence BI. In addition, p-values of each coefficient provide the likelihood that the results did not occur by chance. All the coefficients are statistically highly significant at $p<0.001$, thus the null hypothesis H0: Behavioural intentions (BI) to make water data submissions are not influenced by behavioural attitude (BA), subjective norms (SN) or perceived behavioural control (PBC) is not supported.

\subsection{Motivational factors for participation in crowdsensing for WRM}

The second section of the questionnaire was designed to answer RO2 related to motivational factors for participation in WRM and consisted of closed and open-ended questions. In the closed-ended 
Table 2: Regression analysis with BI as the dependent variable $(n=120)$

\begin{tabular}{lrrrr}
\hline & Coefficients & St. Error & t Stat & P-value \\
\hline BA & 0.43 & 0.09 & 4.55 & 0.00001356 \\
SN & 0.43 & 0.07 & 6.05 & 0.00000002 \\
\hline
\end{tabular}

Table 3: Demotivational factors for submitting data for WRM $(n=120)$

\begin{tabular}{lrr}
\hline Demotivational factor & Frequency & $\%$ \\
\hline Data not being used & 68 & $56.7 \%$ \\
Insufficient time & 55 & $45.8 \%$ \\
Power gap between volunteer and coordinators & 43 & $35.8 \%$ \\
Unappealing recording process & 26 & $21.7 \%$ \\
Lack of confidence & 12 & $10 \%$ \\
Not willing to collect for management purposes & 9 & $7.5 \%$ \\
I do not own a smartphone & 6 & $5 \%$ \\
Volunteer physically unable & 3 & $2.5 \%$ \\
Other & 13 & $10.4 \%$ \\
\hline
\end{tabular}

questions, the respondents were asked to select the three most important motivational factors from a list of all the initial motivational factors in the extended framework of motivational factors (Figure 3). Over two-thirds (72\%) of the selected top factors were from the intrinsic incentives category, 16\% were extrinsic incentives and $12 \%$ were internalised-extrinsic incentives. A large portion (70\%) of the entire population selected at least one factor in the collective incentive category. From these results it can be deduced that intrinsic incentives are the most powerful motivators that encourage the level of participation in crowdsensing for WRM and that collective incentives are the most powerful motivators to participate in WRM.

The three most important motivational factors based on the respondents' responses were: 1) to keep the environment beautiful $(70 \%) ; 2$ ) the respondents feeling responsible to do so $(63.3 \%)$ and 3 ) to contribute to conservation (58.3\%). A checkbox list was also provided in the questionnaire to find out what demotivational factors the respondents perceive affect participation. Table 3 presents the frequency of responses for each demotivational factor.

The most commonly reported demotivational factor was the disbelief the respondents have that the data they submit will actually be used (56.7\%). This disbelief could be due to the lack of responsiveness by service authorities to use generated information to inform their decisions or planning in the WASH sector as reported by (Hellström \& Jacobson, 2014; Minkman, 2015). This lack of responsiveness could also be a reason for the common demotivational factor of feeling a power gap between the respondents and coordinators of such projects (such as municipalities).

In the open-ended questions, respondents were given the option to give other demotivational factors not on the list provided. Thematic analysis (Hellström \& Jacobson, 2014) was used to identify the common themes/factors in the data, resulting in six additional being added as demotivational 
factors to the framework (see Figure 5). These are:

- Long waiting time for issue resolution.

- Lack of flexibility in communication modes.

- Unaware of the monitoring process.

- Unclear on goals and benefits of monitoring.

- Insufficient feedback from project coordinators.

- Not sure if an issue has already been reported.

\begin{tabular}{|c|c|c|}
\hline \multicolumn{2}{|c|}{ INITIAL MOTIVATIONAL FACTORS } & LONG-TERM MOTIVATIONAL FACTORS \\
\hline \multicolumn{2}{|c|}{ INTRINSIC INCENTIVES } & Acknowledgement and Attribution \\
\hline \multirow{2}{*}{$\begin{array}{l}\text { Collective } \\
\text { I feel responsible to do so } \\
\text { Contribute to conservation } \\
\text { Beautiful environment } \\
\text { Teach others } \\
\text { Help others } \\
\text { Help a specific site }\end{array}$} & \multirow{2}{*}{$\begin{array}{l}\text { Intrinsic Motivation } \\
\text { It is fun } \\
\text { I like this project } \\
\text { To kill time } \\
\text { It matches my hobbies } \\
\text { Ability to act independently }\end{array}$} & $\begin{array}{l}\text { Mentorship } \\
\text { Common goals } \\
\text { Trust }\end{array}$ \\
\hline & & $\begin{array}{l}\text { DEMOTIVATIONAL FACTORS } \\
\text { Data not being used }\end{array}$ \\
\hline \multicolumn{2}{|c|}{ INTERNALISED-EXTRINSIC INCENTIVES } & $\begin{array}{l}\text { Lack of confidence } \\
\text { Powrer } \text { gan hatween voluntee }\end{array}$ \\
\hline $\begin{array}{l}\text { Self-Benefit } \\
\text { Learn new skills } \\
\text { Learn new things } \\
\text { Increase chance on a job } \\
\text { Direct feedback } \\
\text { Discover things } \\
\text { Increase my capacity } \\
\text { Get some exercise } \\
\text { Influenced by someone }\end{array}$ & $\begin{array}{l}\text { Self-Efficacy } \\
\text { Contribute to science } \\
\text { Do scientific research }\end{array}$ & $\begin{array}{l}\text { coordinators. } \\
\text { Insufficient time } \\
\text { Volunteer physically unable } \\
\text { Unappealing recording process } \\
\text { Long waiting time for issue resolution. } \\
\text { Lack of flexibility in communication modes. } \\
\text { Unaware of the monitoring process. } \\
\text { Unclear on goals and benefits of monitoring. } \\
\text { Insufficient feedhack from nroiect coordinators }\end{array}$ \\
\hline $\begin{array}{l}\quad \text { EXTRINS } \\
\text { Social Interaction } \\
\text { Do something with friends } \\
\text { Being part of a community } \\
\text { Gain new social contacts } \\
\text { Combine existing skills } \\
\end{array}$ & CENTIVES & Not sure if an issue has already been reported. \\
\hline
\end{tabular}

Figure 5: Updated framework of factors for crowdsensing 


\subsection{Guidelines for crowdsensing projects for WRM}

Based on the findings, seven guidelines (G1 to G7) were identified that can assist with project planning and coordination of crowdsensing projects for WRM.

G1: Provide clarity on goals and results. Clarity on the project goals, data submissions and analyses must be regularly communicated so as to create more positive attitudes towards making submissions, as this will make citizens aware of the importance of their submissions for water resources in their environment.

G2: Ensure flexible communication modes. Crowdsensing systems should allow users to submit multiple types of data sources (such as images, video and audio) to allow the provision of supplemental and more accurate information and to generate a holistic view of the point of interest. However, multiple resources may contribute conflicting information about the same object. One method to help this is truth discovery, which can aggregate noisy data to estimate the reliability of each source (Guo et al., 2014; Lowry \& Fienen, 2013), and to solve various issues such as data integration and truth detection (Lowry \& Fienen, 2013).

G3: Use simple data collection protocols. The complexity of data collection protocols affects the engagement of participants in crowdsensing projects and may result in fewer participants. Simplified protocols do not only improve the appeal of submitting data but also minimise the learning curve of the monitoring process and the time needed to submit data. However, these protocols must still ensure the collection of high-quality data and protect the confidentiality and privacy of participants.

G4: Incorporate different types of incentives. Collecting data for the good of the community or environment might not be enough to motivate citizens to participate in crowdsensing. Thus, other incentives from the other incentive categories (self-benefit, self-efficacy, intrinsic incentives and social interraction) should be incorporated.

G5: Implement effective feedback mechanisms. Participants want to know if an issue has already been reported and the progress of the issue resolution. Most crowdsensing systems require instant answers, thus, there is a need for fast and efficient systems to provide access to, retrieval of and processing of the data. Such systems will enable decision makers to provide instant feedback or react fast to inform the public about upcoming events and/or organise major actions to protect the citizens. There is a need to aggregate robust machine learning algorithms and decision algorithms into a computing framework that can analyse large data sets to find meaningful domain-specific insights from the data and make the right decision when analysis shows certain behaviours and findings (Yang et al., 2015).

G6: Promote community pro-environmental behavioural change. An individual's perceptions of subjective norms have a significant influence on the motivation to comply with those pressures and subjective norms play a very important role in shaping behaviours. Seeing others in an individual's immediate environment making water data submissions increases the chance 
of the individual performing the behaviour. Thus, a critical success factor for crowdsensing is to develop a social marketing approach that promotes pro-environmental behaviour in communities.

G7: Use participant retention strategies (both short term and long term). It is important to develop strategies on how to retain participants in crowdsensing projects as it is more cost-effective to retain a participant than acquire a new one. It is necessary to ensure the participants derive benefits from information sharing (Pankratius et al., 2014) to ensure continued motivation and retention of participants. If participants believe making data submissions is useful, their BA will be positive, hence increasing the chance of their continued submissions. Strategies can include effective feedback mechanisms (G5), updates on the progress of issue resolution (G1) and notification alerts of contamination events detected close to users' location.

\section{CONCLUSIONS}

This paper provides a valuable contribution to both practitioners and researchers. The comprehensive framework of motivational and demotivational factors for crowdsensing can be used by crowdsensing project coordinators as well as researchers in the field of crowdsensing. The framework considers the initial as well as the long-term motivational factors for crowdsensing, and these are classified according to the type of motivation that encourages participation. The findings provide the opportunity for a more in depth understanding of citizen profiles of citizens who can potentially participate in crowdsensing for WRM. The incentive theory and TPB theory were successfully used to provide the lens for deriving the framework and conducting the survey. The regression analysis of the survey data did not support the null hypothesis that an individual's Behavioural intentions (BI) to make water data submissions are not influenced by behavioural attitude (BA), subjective norms (SN) or perceived behavioural control (PBC). The findings also revealed that citizens would participate in WRM for the common good. However, the three most frequently cited factors that can demotivate citizens to participate were disbelief in the usage of the submitted data, insufficient time to make submissions and the negative power relations between citizens and the project coordinators.

To increase the chances of buy in to participation and technology acceptance, this study highlights the importance of considering the potential participant profile and understanding the social aspects and motivational factors of crowdsensing. Several guidelines for crowdsensing are made based on the responses from citizens. The framework and guidelines proposed in this paper can add value to crowdsensing projects since they can assist with the planning and design of crowdsensing projects and systems.

The findings from this research were used in the larger research study to design a crowdsensing method for successful WRM in smart communities. Due to space constraints the details of this method could not be included in the paper and will be reported on in future work. Other future work could include the implementation of this framework using a larger sample population. Future research could also conduct an analysis to find patterns that lead to the success or failure of crowdsensing projects and to determine the impact of incorporating the guidelines made in this paper on the 
success of crowdsensing projects in WRM.

\section{References}

Ajzen, I. (1991). The theory of planned behavior. Organizational behavior and human decision processes, 50(1), 179-211. https://doi.org/10.1016/0749-5978(91)90020-T

Baltar, F. \& Brunet, I. (2012). Social research 2.0: Virtual snowball sampling method using Facebook. Internet research, 22(1), 57-74. https://doi.org/10.1108/10662241211199960

Bosha, E., Cilliers, L. \& Flowerday, S. (2017). Incentive theory for a participatory crowdsourcing project in a developing country. South African journal of information management, 19(1). https://doi.org/10.4102/sajim.v19i1.739

Bozzon, A., Houtkamp, J., Kresin, F., de Sena, N. \& de Weerdt, M. (2015). From needs to knowledge: A reference framework for smart citizens initiatives. Last accessed 09 Dec 2019. Retrieved from https://www.ams-amsterdam.com/wordpress/wp-content/uploads/2016/10/ProjectFinal-Reporting-From-needs-to-knowledge.pdf

Braun, V. \& Clarke, V. (2006). Using thematic analysis in psychology. Qualitative research in psychology, 3(2), 77-101. https://doi.org/10.1191/1478088706qp063oa

Cilliers, L., Flowerday, S. \& McLean, S. (2016). A crowdsourcing, smart city model for a developing country. In CONF-IRM 2016 Proceedings.

de Montalvo, U. (2003). Mapping the determinants of spatial data sharing. https://doi.org/10. 4324/9781315197203

Economic and Social Council. (2018). Progress towards the sustainable development goals. International organization, 1(1), 103-115. https://doi.org/10.1017/S0020818300006640

Fishbein, M. \& Ajzen, I. [Icek]. (2011). Predicting and changing behavior: The reasoned action approach. Taylor \& Francis.

Gao, J., Li, Q., Zhao, B., Fan, W. \& Han, J. (2015). Truth discovery and crowdsourcing aggregation. In Proceedings of the VLDB Endowment - Proceedings of the 41st International Conference on Very Large Data Bases (pp. 2048-2049). https://doi.org/10.14778/2824032.2824136

Gassenheimer, J., Siguaw, J. \& Hunter, G. (2013). Exploring motivations and the capacity for business crowdsourcing. AMS review, 3(4). https://doi.org/10.1007/s13162-013-0055-8

Geoghegan, H., Dyke, A., Pateman, R., West, S. \& Everett, G. (2016). Understanding motivations for citizen science. Final report on behalf of the UK Environmental Observation Framework. Last accessed 09 Dec 2019. Retrieved from http://www.ukeof.org.uk/resources/citizen-scienceresources/MotivationsforCSREPORTFINALMay2016.pdf

Gharesifard, M. \& Wehn, U. (2015). To share or not to share: Drivers and barriers for sharing data via online amateur weather network. Journal of hydrology, 535(1), 181-190. https: //doi.org/10.1016/j.jhydrol.2016.01.036

Glotzbach, R., Barakzai, S., Adisu, J. \& Tiwari, C. (2013). Functionality: The challenge to sustain rural water supply services. Last accessed 09 Dec 2019. Retrieved from http://www. improveinternational.org/wp-content/uploads/2016/04/snv_wash_functionality_ practice_brief_october_2013_0.pdf 
Gong, X. \& Shroff, N. (2017). Truthful mobile crowdsensing for strategic users with private qualities. In 15th International Symposium on Modeling and Optimization in Mobile, Ad Hoc and Wireless Networks (WiOpt). https://dx.doi.org/10.23919/WIOPT.2017.7959903

Guo, B., Yu, Z., Zhou, X. \& Zhang, D. (2014). From participatory sensing to mobile crowd sensing. In IEEE International Conference on Pervasive Computing and Communication Workshops (pp. 593598). https://doi.org/10.1109/PerComW.2014.6815273

Hall, N., Jaramillo, C. A., Jagals, P., Currie, D., Ossa-Moreno, J., Dean, A., ... Lopez, D. C. (2016). Strengthening community participation in meeting UN Sustainable Development Goal 6 for water, sanitation and hygiene. Global Change Institute, the University of Queensland, Brisbane.

Han, K., Graham, E., Vassallo, D. \& Estrin, D. (2011). Enhancing motivation in a mobile participatory sensing project through gaming. In IEEE International Conference on Privacy, Security, Risk and Trust and IEEE International Conference on Social Computing (pp. 1443-1448). https: //doi.org/10.1109/PASSAT/SocialCom.2011.113

Harpe, S. (2015). How to analyze likert and other rating scale data. Currents in pharmacy teaching and learning, 7(6), 836-850. https://doi.org/10.1016/j.cptl.2015.08.001

He, D., Chan, S. \& Guizani, M. (2015). User privacy and data trustworthiness in mobile crowd sensing. IEEE wireless communications, 22(1), 28-34. https://doi.org/10.1109/MWC.2015.7054716

Hellström, J. \& Jacobson, M. (2014). 'you can't cheat the community anymore'-using mobiles to improve water governance. In Proceedings of 4th International Conference on M4D Mobile Communication for Development (pp. 48-59).

Hung, N. Q. V., Tam, N., Tran, L. \& Aberer, K. (2013). An evaluation of aggregation techniques in crowdsourcing. In Web Information Systems Engineering-WISE 2013 (pp. 1-15). https: //doi.org/10.1007/978-3-642-41154-0_1

Hutchings, M., Dev, A., Palaniappan, M., Srinivasan, V., Ramanathan, N. \& Taylor, J. (2012). mWASH: Mobile phone applications for the water, sanitation, and hygiene sector. Last accessed 09 Dec 2019. Retrieved from https://pacinst.org/publication/mwash-mobile-phone-applicationsfor-the-water-sanitation-and-hygiene-sector/

Jaimes, L., Vergara-Laurens, I. \& Raij, A. (2015). A survey of incentive techniques for mobile crowd sensing. IEEE Internet of Things journal, 2(5), 370-380. https://dx.doi.org/10.1109/JIOT. 2015.2409151

Jiménez, A. \& Pérez-Foguet, A. (2010). Challenges for water governance in rural water supply: Lessons learned from Tanzania. International journal for water resources development, 26(2), 235-248. https://doi.org/10.1080/07900621003775763

Joachim, O., Kamarudin, N., Aliagha, G. \& Ufere, K. (2015). Theoretical explanations of environmental motivations and expectations of clients on green building demand and investment. In IOP Conference Series: Earth and Environmental Science Volume 23 Conference 1. https://doi.org/ 10.1088/1755-1315/23/1/012010

Kanhere, S. (2011). Participatory sensing: Crowdsourcing data from mobile smartphones in urban spaces. In IEEE 12th International Conference on Mobile Data Management (pp. 3-6). https: //dx.doi.org/10.1007/978-3-642-36071-8-2 
Killeen, P. (1982). Incentive theory: II. Models of choice. Journal of the experimental analysis of behavior, 38(2), 217-232. https://doi.org/10.1901/jeab.1982.38-217

Larose, D. (2006). Data mining methods and models. John Wiley \& Sons.

Lendák, I. (2016). Mobile crowd-sensing in the Smart City. In C. Capineri, M. Haklay, H. Huang, V. Antoniou, J. Kettunen, F. Ostermann \& R. Purves (Eds.), European handbook of crowdsourced geographic information.

Liu, J., Shen, H. \& Zhang, X. (2016). A survey of mobile crowdsensing techniques: A critical component for the internet of things. In International Conference on Computer Communication and Networks. https://doi.org/10.1109/ICCCN.2016.7568484

Lowry, C. \& Fienen, M. (2013). CrowdHydrology: Crowdsourcing hydrologic data and engaging citizen scientists. GroundWater, 51 (1), 151-156. https://doi.org/10.1111/j.1745-6584. 2012.00956.x

Martin, V., Weiler, B., Reis, A., Dimmock, K. \& Scherrer, P. (2017). 'doing the right thing': How social science can help foster pro-environmental behaviour change in marine protected areas. Marine policy, 81(4). https://doi.org/10.1016/j.marpol.2017.04.001

Massung, E., Coyle, D., Cater, K., Jay, M. \& Preist, C. (2013). Using crowdsourcing to support proenvironmental community activism. In Proceedings of the SIGCHI Conference on Human Factors in Computing Systems (pp. 371-380). https://doi.org/10.1145/2470654.2470708

Minkman, E. (2015). Citizen science in water quality monitoring (Master's thesis, Delft University of Technology, Delft, Holland).

Nel, P., Booysen, M. \& van der Merwe, B. (2014). ICT-enabled solutions for smart management of water supply in Africa. In Proceedings of the First International Conference on the use of Mobile Informations and Communication Technology (ICT) in Africa UMICTA 2014 (pp. 40-44).

Nevid, J. (2013). Psychology: Concepts and applications. Wadsworth Cengage learning.

Nique, M. \& Opala, K. (2014). The synergies between mobile, energy and water access: Africa. Last accessed 09 Dec 2019. Retrieved from https://www.gsma.com/mobilefordevelopment/ resources/the-synergies-between-mobile-energy-and-water-access-africa/

Ogie, R. (2016). Adopting incentive mechanisms for large-scale participation in mobile crowdsensing: From literature review to a conceptual framework. Human-centric computing and information sciences, 6(24). https://doi.org/10.1186/s13673-016-0080-3

Pankratius, V., Lind, F., Coster, A., Erickson, P. \& Semeter, J. (2014). Mobile crowd sensing in space weather monitoring: The Mahali project. IEEE communications magazine, 52(8), 22-28. https: //doi.org/10.1109/MCOM.2014.6871665

Rivett, U., Gool, S., Champanis, M. \& Nyemba-Mudenda, M. (2014). ICTs in the water sector-where do we stand? Last accessed 09 Dec 2019. Retrieved from https://www.researchgate.net/ publication/277203934_ICTs_in_the_Water_Sector_-_where_do_we_stand

Rotman, D., Hammock, J., Preece, J., Hansen, D. \& Boston, C. (2014). Motivations affecting initial and long-term participation in citizen science projects in three countries. In iConference 2014 Proceedings (pp. 110-124). https://doi.org/10.9776/14054 
Rutten, M., Minkman, E. \& van der Sanden, M. (2017). How to get and keep citizens involved in mobile crowd sensing for water management? A review of key success factors and motivational aspects. Wiley Interdisciplinary Review of Water, 4. https://dx.doi.org/10.1002/wat2.1218

Sarstedt, M., Ringle, C., Smith, D., Reams, R. \& Hair, J. (2014). Partial least squares structural equation modeling (PLS-SEM): A useful tool for family business researchers. Journal of family business strategy, 5(1), 105-115. https://doi.org/10.1016/j.jfbs.2014.01.002

Traverso, P. (2015). Keynote abstract: From smart cities to smart communities: The case of children's independent mobility. In 10th International Workshop on Semantic and Social Media Adaptation and Personalisation (p. xv).

Wiggins, A. (2011). eBirding: Technology adoption and the transformation of leisure into science. In Proceedings of the 2011 iConference (pp. 798-799). https://doi.org/10.1145/1940761. 1940910

Wilson, P. (1997). Smart communities guidebook. State University of San Diego.

Yadav, K., Chakraborty, D., Soubam, S., Prathapaneni, N., Nandakumar, V., Naik, V., ... De, P. (2013). Human sensors: Case study of open-ended community sensing in developing regions. In Proceedings of the IEEE International Conference on Pervasive Computing and Communications Workshops (PerCom '13) (pp. 389-392).

Yang, D., Xue, G., Fang, X. \& Tang, J. (2015). Incentive mechanisms for crowdsensing: Crowdsourcing with smartphones. IEE/ACM transactions on networking, 24(3), 1-13. 